\title{
Neural Mechanisms Underlying Risk and Ambiguity Attitudes
}

\author{
Neeltje E. Blankenstein, Jiska S. Peper, Eveline A. Crone, \\ and Anna C. K. van Duijvenvoorde
}

\begin{abstract}
Individual differences in attitudes to risk (a taste for risk, known probabilities) and ambiguity (a tolerance for uncertainty, unknown probabilities) differentially influence risky decisionmaking. However, it is not well understood whether risk and ambiguity are coded differently within individuals. Here, we tested whether individual differences in risk and ambiguity attitudes were reflected in distinct neural correlates during choice and outcome processing of risky and ambiguous gambles. To these ends, we developed a neuroimaging task in which participants $(n=50)$ chose between a sure gain and a gamble, which was either risky or ambiguous, and presented decision outcomes (gains, no gains). From a separate task in which the amount, probability, and ambiguity level were varied, we estimated individuals' risk and ambiguity attitudes. Although there was pronounced neural over-
\end{abstract}

\section{INTRODUCTION}

Many of our decisions are characterized by an element of risk, that is, an uncertainty in the outcomes we might encounter. For instance, we may place a bet in a game of roulette hoping to win large amounts of money (with the risk of losing money), or we may smoke with the risk of developing cancer. However, a fundamental difference in these types of risk is that, in the former case, the probabilities of the possible outcomes are known (e.g., the chance of winning in roulettes when betting on the color black is slightly less than 50\%), whereas in the latter case, the probabilities are unknown (e.g., one does not know the exact chance of developing cancer). This distinction between known and unknown risks has long been acknowledged in the decision-making literature as "explicit" risk and "ambiguous" risk, respectively (Tversky \& Kahneman, 1992; Knight, 1921; henceforth referred to as risk and ambiguity). Classic behavioral experiments have shown that risk and ambiguity are distinct types of uncertainty that both influence our choice behavior (Ellsberg, 1961). That is, although, generally, people are both averse to risk and ambiguity and show a stronger aversion to ambiguity than risk alone, individuals' prefer-

Leiden University lap between risky and ambiguous gambling in a network typically related to decision-making under uncertainty, relatively more risk-seeking attitudes were associated with increased activation in valuation regions of the brain (medial and lateral OFC), whereas relatively more ambiguity-seeking attitudes were related to temporal cortex activation. In addition, although striatum activation was observed during reward processing irrespective of a prior risky or ambiguous gamble, reward processing after an ambiguous gamble resulted in enhanced dorsomedial PFC activation, possibly functioning as a general signal of uncertainty coding. These findings suggest that different neural mechanisms reflect individual differences in risk and ambiguity attitudes and that risk and ambiguity may impact overt risk-taking behavior in different ways. ences for risk and ambiguity are often uncorrelated (Von Gaudecker, Van Soest, \& Wengström, 2011; Ellsberg, 1961). Although prior studies have examined the neural mechanisms underlying these distinct types of uncertainty, it is not yet well understood if risk and ambiguity at the neural level can be disentangled within individuals.

In general, two main brain systems have been implicated during decision-making (for a review, see Platt \& Huettel, 2008). First, a system that responds to reward contingencies has been related to the ventral striatum (VS) and ventral medial PFC/OFC (Bartra, McGuire, \& Kable, 2013; Levy \& Glimcher, 2012; Knutson, Taylor, Kaufman, Peterson, \& Glover, 2005; Kuhnen \& Knutson, 2005; O’Doherty, Kringelbach, Rolls, Hornak, \& Andrews, 2001). This valuation system may reflect the subjective (Levy, Snell, Nelson, Rustichini, \& Glimcher, 2010) or objective (van Duijvenvoorde et al., 2015) expected value (EV) of the choice at hand but is also related to processing rewarding outcomes (Delgado, Nystrom, Fissell, Noll, \& Fiez, 2000) and producing learning signals (O'Doherty, 2004). A second system, including the insular cortex, lateral PFC, dorsolateral PFC, and posterior parietal cortex (PPC) is more central to the evaluation of the uncertainty of choice options (Levy, 2016; Mohr, Biele, \& Heekeren, 2010; Platt \& Huettel, 2008) with the PPC being particularly important for assessing probabilities (Huettel, Song, \& McCarthy, 
2005). Thus, although a system of subcortical (VS) and cortical (medial PFC) regions appears to be responsible for choice valuation and reward learning, a cortical system (insula, lateral PFC, PPC) may be more related to executive, computational processes in risky decisions, such as assessing uncertainty.

Several prior neuroimaging studies have tested for associations between decisions under risk and/or ambiguity and brain activation. That is, one study that compared risky and ambiguous gambling observed increased activation for ambiguity compared with risk in the amygdala and medial OFC, whereas risk compared with ambiguity elicited more activation in the striatum (Hsu, Bhatt, Adolphs, Tranel, \& Camerer, 2005). However, another study observed increased activation for ambiguity compared with risk in the insula, lateral PFC, and PPC (Huettel, Stowe, Gordon, Warner, \& Platt, 2006). Both studies concluded that these brain regions are responsible for resolving uncertainty. Yet, similar patterns of activation for both risk and ambiguity have also been observed, with overlapping activation in the medial PFC, PPC, amygdala, and striatum (Levy et al., 2010). Thus, although these studies found areas of activation typical for decision-making (i.e., valuation and uncertainty coding), it is not yet well understood whether risky and ambiguous decision-making rely on distinct neural mechanisms.

A valuable addition to studying the neural specificity of risk and ambiguity processing may be to include individuals' preferences, that is, attitudes, toward uncertainty. Whereas someone's risk attitude reflects to what extent one makes a trade-off between outcome magnitudes (e.g., the size of a monetary reward) against the probability of that outcome, ambiguity attitude reflects how one deals with the uncertainty around outcome probabilities (i.e., pessimistic or optimistic about the unknown probabilities; e.g., see Levy, 2016). An elegant way to estimate these preferences is by formally modeling risk and ambiguity attitudes from tasks in which the gain probability, gain amount, and level of ambiguity are varied. Behaviorally, this model-based method has been applied successfully in developmental samples (Blankenstein, Crone, van den Bos, \& van Duijvenvoorde, 2016; Tymula et al., 2012) as well as in adults (Tymula, Rosenberg Belmaker, Ruderman, Glimcher, \& Levy, 2013) and provides a sensitive measure of someone's risk and ambiguity preferences.

Several studies have started to relate individuals' risk or ambiguity attitudes to neural activity in decision-making. For instance, a number of studies observed that greater risk aversion was related to greater activation in inferior frontal gyrus, lateral PFC, and lateral OFC, both with model-based estimations of risk aversion (Christopoulos, Tobler, Bossaerts, Dolan, \& Schultz, 2009; Tobler, O'Doherty, Dolan, \& Schultz, 2007) as well as with model-free risk-averse behavior (Fecteau et al., 2007; Knoch et al., 2006). Contrary, a greater risk-seeking attitude has also been positively related to activation in the lateral OFC, ventromedial PFC, and PPC (Engelmann \&
Tamir, 2009; Tobler et al., 2007; Huettel et al., 2006). Individual differences in ambiguity attitude have been studied to a lesser extent. Some studies revealed that more ambiguity aversion was related to increased activation in lateral OFC (Hsu et al., 2005) and medial PFC (Pushkarskaya, Smithson, Joseph, Corbly, \& Levy, 2015), whereas another study found greater activation in a neighboring region (ventrolateral PFC) with more ambiguity-seeking attitudes (Huettel et al., 2006). These previous studies, carried out in relatively small samples (e.g., $n=16$, Tobler et al., 2007; $n=10$, Engelmann \& Tamir, 2009; $n=16$, Hsu et al., 2005; $n=13$, Huettel et al., 2006), thus show conflicting findings and did not yet disentangle risk and ambiguity attitudes within individuals.

In the current study, we aimed to examine the neural correlates of decision-making under risk and ambiguity and study the association with individual differences in risk and ambiguity attitudes in a sample of 50 healthy adults (a recommended minimal sample size for analyses of individual differences [Yarkoni, Poldrack, Van Essen, \& Wager, 2010; Yarkoni, 2009]). To get a robust measure of neural activation during risky and ambiguous gambling and subsequent outcome processing, we administered a straightforward monetary gambling task. Here, participants chose between a consistent sure gain and a gambling option, which was either risky or completely ambiguous, and presented subsequent reward outcomes (gain or no gain). We were particularly interested in the neural response during an active gamble, because previous studies have shown that decision-making and subsequent reward processing are more robust when an active choice is made rather than passively viewing the stimuli (Studer, Apergis-Schoute, Robbins, \& Clark, 2012; Rao, Korczykowski, Pluta, Hoang, \& Detre, 2008; Tricomi, Delgado, \& Fiez, 2004).

To derive risk and ambiguity attitudes, we modeled each individual's risk and ambiguity attitudes from a separate behavioral task administered after the MRI session (see also Blankenstein et al., 2016; modeled after Tymula et al., 2012, 2013). This enabled us to investigate the relation between risk and ambiguity attitudes and brain activation during risky and ambiguous gambling. Although prior findings are mixed, one region that has relatively consistently been associated with risk attitude is the OFC/medial PFC (e.g., Engelmann \& Tamir, 2009; Tobler et al., 2007). In the current study, we therefore expected that individual differences in risk attitude would be associated with neural activation in the OFC/medial PFC during risky gambling. Alternatively, risk attitudes may correlate more specifically with neural activation related to assessing uncertainty and probabilities such as the lateral PFC and PPC (Christopoulos et al., 2009; Fecteau et al., 2007; Huettel et al., 2006; Knoch et al., 2006). Fewer studies have investigated relations between ambiguity attitude and brain activation (Pushkarskaya et al., 2015; Huettel et al., 2006; Hsu et al., 2005). A central hypothesis based on this prior work would be that 
individual's tendency to seek out ambiguity is related to control regions in the brain such as the lateral PFC (Huettel et al., 2006).

Furthermore, the association between risk versus ambiguity within a choice at hand and processing subsequent reward outcomes (gain or no gain) has yet to be examined. That is, although it is well known that processing rewards is related to increased activation in the VS and medial PFC (e.g., see Delgado et al., 2000), to our knowledge, no study to date has explicitly disentangled reward processing after a risky gamble from reward processing after an ambiguous gamble. Given that behavior in response to risk and ambiguity differs (Von Gaudecker et al., 2011; Ellsberg, 1961), it is possible that processing rewards after risk and ambiguity yields different responses in the reward circuitry of the brain as well. Thus, in addition, we explored whether processing rewards after risk or ambiguity would yield differential activation in regions typically associated with reward (i.e., VS and ventral medial PFC) and whether this differential rewardrelated activation was associated with individual differences in risk and ambiguity attitudes.

\section{METHODS}

\section{Participants}

Fifty-seven participants (30 women) between 18 and 28 years old took part in this study. All participants were recruited via local advertisements in the Netherlands and provided written informed consent. This study was approved by the institutional review board of the Leiden University Medical Centre. All anatomical scans were cleared by a radiologist, and no abnormalities were reported. Participants were screened for MRI contraindications and neurological or psychiatric disorders, had normal or corrected-to-normal vision, and were righthanded. Five participants reported to have been diagnosed with a disorder, including depression, anxiety, attention deficit hyperactivity disorder, and cyclothymic disorder (a mild form of bipolar disorder). These participants were scanned but were excluded from all analyses. Note that, when we reran our analyses including these participants, this did not qualitatively affect our results. In addition, one participant was excluded because of too few trials in which the gambling option was chosen (i.e., <10 gambles), and one participant was excluded because of excessive head motion in the scanner (i.e., $>3 \mathrm{~mm}$ ). The final sample therefore included 50 healthy participants ( 25 women; $M_{\text {age }}=23.71$ years, $S D_{\text {age }}=2.56$, age range $=18.85-28.46$ years $)$.

\section{Wheel of Fortune Task}

\section{fMRI Task}

Participants played a wheel of fortune task (Figure 1) in which they were asked to make a number of choices

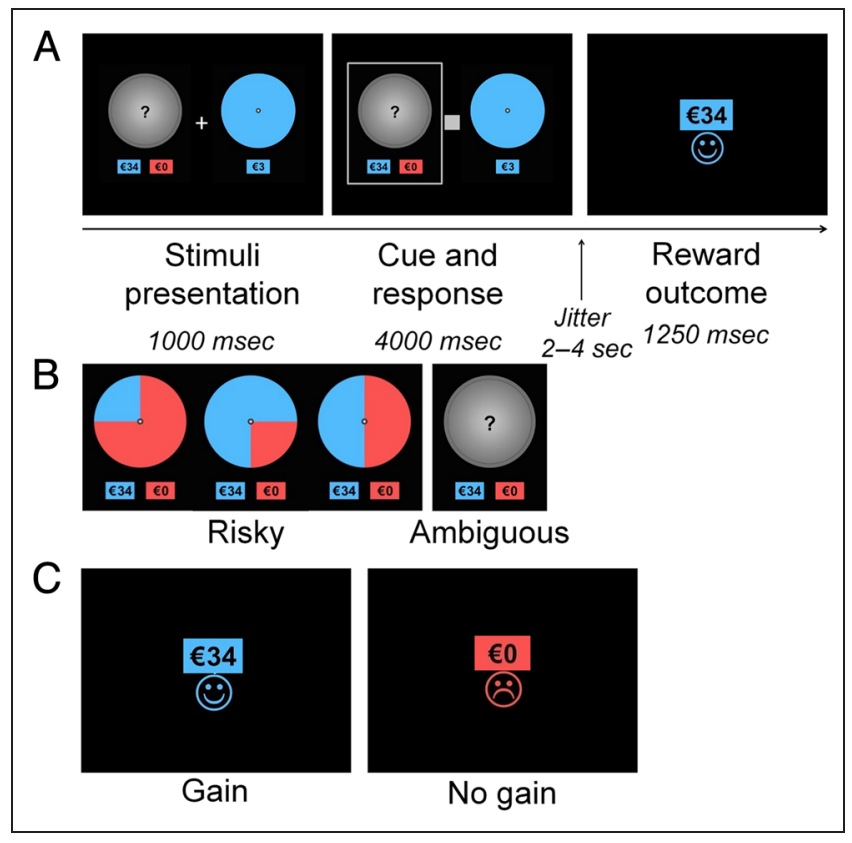

Figure 1. (A) Example of the trial sequence of the fMRI task showing an ambiguous trial with gain as reward outcome. Each trial started with a 500-msec fixation cross, after which the wheels appeared. After $1000 \mathrm{msec}$, a gray square appeared in the center of the screen prompting the participants to respond. A response had to be given within $3000 \mathrm{msec}$. A gray selection frame confirmed the participant's choice. After a jittered fixation cross (2000-4000 msec with increments of $500 \mathrm{msec}$ ), participants were presented with the reward outcome of their choice (gain or no gain), which was visible for $1250 \mathrm{msec}$. The next trial began after an intertrial interval with intervals varying between 0 and $9350 \mathrm{msec}$ (jittered). (B) The different gambling wheels. (C) Gain and no gain outcomes.

between pairs of wheels, presenting a safe (a consistent sure gain of $€ 3$ ) versus a gambling option, which could yield more money ( $€ 31, € 32$, $€ 33$, or $€ 34$; varied to keep participants engaged in the task) but could also yield nothing $(€ 0)$. The gambling wheel presented either a risky gamble or an ambiguous gamble. In the risky gambles, the probabilities were known, with blue indicating the portion of the wheel corresponding to gain and red indicating the portion of the wheel corresponding to no gain. In the ambiguous gambles, the probabilities were completely hidden by a gray "lid" with a question mark on it. Participants played 92 trials: 46 ambiguous trials and 46 risky trials, which were presented intermixed. Of the risky trials, 30 trials reflected a gamble with a 50\% gain probability, 8 trials reflected a gamble with a $75 \%$ gain probability, and 8 trials reflected a gamble with a 25\% gain probability (Figure 1B).

After the choice, participants were presented with reward feedback (gain or no gain; Figure 1C). This was done to investigate effects of risk and ambiguity within the choice at hand on subsequent reward processing and to study potential effects of risk and ambiguity attitudes on reward processing after a risky or ambiguous gamble. We programmed the experiment such that the 
probabilities presented in the wheels $(25 \%, 50 \%$, or $75 \%)$ matched the actual possibilities of winning when choosing to gamble. For example, for the 50\% risk trials, there was a $50 \%$ chance of winning when choosing the gamble. That is, the computer, on a trial-by-trial basis, randomly selected (without replacement) either gain or no gain in half of the trials. The order of gains and no gains was randomized for each participant. We observed that, on average, participants' experienced probabilities in risky and ambiguous gambles matched the presented probabilities. Finally, on each trial, the computer randomly selected (without replacement) one of the four possible amounts ( $€ 31, € 32$, $€ 33$, or $€ 34$ ) to display on a trialby-trial basis. Thus, although each individual was exposed to the same distribution of probabilities, the amount varied per trial. Reward feedback for gains was presented as the amount in blue. Reward feedback for no gains was presented as $€ 0$ in red. To motivate participants to frequently choose the gamble option, the EV (the amount of a choice option multiplied by its probability) of the gamble option was considerably higher than the EV of the safe option (which was always $€ 3$ ). This enabled the comparison of brain activation during gambling under risk with brain activation during gambling under ambiguity and during their corresponding outcomes.

The task was presented in the MRI scanner via E-prime (Psychology Software Tools, Pittsburgh, PA) and started with a 500-msec fixation cross, after which the wheels appeared. After $1000 \mathrm{msec}$, a gray square appeared in the center of the screen cuing the participants to respond. Responses had to be given within a 3000-msec interval. Participants responded to the task with their right index finger (to select the wheel on the left) and right middle finger (to select the wheel on the right). After the response was made, a gray selection frame appeared around the chosen wheel, confirming the participant's choice. This remained visible for the duration of the 3000-msec interval. In case of no response, the words "TOO LATE" appeared in the center of the screen for $1250 \mathrm{msec}$, after which the next trial began. This happened only in $0.67 \%$ of the trials, and these trials were excluded from all analyses. A jittered fixation cross (2000-4000 msec with increments of $500 \mathrm{msec}$ ) separated the choice phase from the outcome phase. The reward outcomes (gain, no gain, or safe gain) were presented for $1250 \mathrm{msec}$. The optimal trial sequence (i.e., the ordering of risky and ambiguous trials) and the intertrial intervals were chosen using OptSeq (Dale, 1999), with jittered intervals varying between 0 and $9350 \mathrm{msec}(M=1961 \mathrm{msec})$. The 500-msec fixation cross that preceded each trial was not part of the intertrial interval.

Finally, we randomly displayed the different wheels (gamble, safe) left and right on the screen. In addition, the risky wheels had varying color configurations that were presented randomly on a trial-by-trial basis, with the blue proportion of the wheel displayed in the left or right portion of the wheel (in the case of 50\% probability trials) or the upper left, upper right, lower left, or lower right portion of the wheel (in the case of $25 \%$ and $75 \%$ probability trials).

\section{Behavioral Task}

To scrutinize individuals' risk and ambiguity attitudes, a behavioral version of the wheel of fortune task was administered after the fMRI session as validated previously (Blankenstein et al., 2016) and modeled after Tymula et al. (2012). To derive sensitive measures of risk and ambiguity attitudes from individuals that could not be influenced by changes in the decision environment, no outcomes were provided in this task.

In this behavioral task, the gambling wheel varied in amount ( $€ 5$, $€ 8$, $€ 20$, or $€ 50$ ), probability ( 0.125 , $0.25,0.375,0.50,0.625$, or 0.75$)$, and ambiguity level (0\%, $25 \%, 50 \%, 75 \%$, or $100 \%)$. The level of ambiguity was manipulated by varying the size of the "lid" covering the wheel. Combining all amounts with all probabilities resulted in 24 unique risk trials. Combining all amounts with all ambiguity levels resulted in 16 unique ambiguous trials. All trials were presented twice, resulting in 80 trials, which were used to estimate individuals' risk and ambiguity attitudes (see Model-based Estimations of Risk and Ambiguity Attitudes section).

The task was presented after the fMRI session via E-prime (Psychology Software Tools). Each trial started with a jittered fixation cross (between 500 and $1000 \mathrm{msec}$, with increments of $100 \mathrm{msec}$ ) after which the wheels appeared. After $1000 \mathrm{msec}$, a gray square appeared in the center of the screen, prompting the participants to respond using their right index finger (left wheel) and middle finger (right wheel). Response time was selfpaced. A yellow selection frame confirmed the participant's choice $(500 \mathrm{msec})$. Similar to the fMRI task, we controlled for effects of attention and key preference by counterbalancing the position of the blue and red parts of the wheel (left, right, bottom, and top of the wheel) and the position of the ambiguous lids (top or bottom) across trials. Finally, the different wheels (gamble, safe) were randomly displayed left and right on the screen.

\section{Procedure}

Participants received instructions on the MRI session in a quiet laboratory room. Next, the wheel of fortune fMRI task was explained. Participants were instructed that the ambiguous wheel could reflect a gamble of any of the risky probabilities $(25 \%, 50 \%, 75 \%)$, and they practiced 10 trials on a laptop. Participants were told that, after the task, the computer would randomly select the outcomes of three trials, of which the average amount was paid out in addition to a standard payout fee. Eventually, the computer randomly selected a rounded average of a gain trial, a no gain trial, and a safe gain trial, which 
amounted to an additional payout of $€ 11$ or $€ 12$. The wheel of fortune task was presented in two runs of 9 min each, with a short break in between. Stimuli were presented on a screen, which was visible via a mirror that was placed on the head coil. Participants responded to the task with their right index finger (to select the wheel on the left) and right middle finger (to select the wheel on the right) using a button box that was attached to the participant's leg. Head movements were restricted by inserting foam padding between the participant's head and the head coil. After the MRI session, participants completed the behavioral version of the wheel of fortune task, which lasted approximately 20 min. Here, participants were given a hypothetical choice task and instructed to choose their preferred option. We explained the different levels of ambiguity by showing the different "lids" that could vary in size and cover more or less of the wheel and show the wheels that could lie underneath these lids. Participants played three practice trials before the task began.

\section{Model-based Estimations of Risk and Ambiguity Attitudes}

To estimate each participant's risk and ambiguity attitudes from the behavioral task, we modeled the subjective value (or expected utility [EU]) of each choice option by using a power utility function with an additional term to take into account ambiguity attitude (Blankenstein et al., 2016; Tymula et al., 2012; Levy et al., 2010; Gilboa \& Schmeidler, 1989):

$$
E U(x, p, A)=\left(p-\beta \times \frac{A}{2}\right) \times x^{\alpha}
$$

In this equation, $x$ denotes the amount, $p$ denotes the probability, $A$ denotes the ambiguity level, $\alpha$ denotes the risk attitude, and $\beta$ denotes the ambiguity attitude. An $\alpha$ of 1 indicates a purely linear utility function, indicating a risk-neutral attitude. An $\alpha<1$ indicates a concave utility function and thus a risk-averse attitude. Conversely, an $\alpha>1$ indicates convexity and thus a risk-seeking attitude. To assess subjective value, we multiplied the utility of a choice option with the probability of the (hypothetical) outcome. Here, the ambiguity level was taken into account, with $p$ as the objective probability, $\beta$ as the individual ambiguity attitude to be estimated, and $A$ as the objective ambiguity level. A $\beta$ of 0 indicates an ambiguity-neutral attitude, meaning that the individual is unaffected by the level of ambiguity. A $\beta>0$ indicates an ambiguity-averse attitude, meaning that the individual would behave as if the probability is less than the objective probability (50\%). Finally, a $\beta<1$ would indicate an ambiguity-seeking attitude, in which case the individual would behave as if the probability is more than the objective probability.
For model fitting, the simplex algorithm of the general purpose optimization toolbox (optim) in $\mathrm{R}$ was used (R Core Team, 2015). To model trial-by-trial choices, a logistic choice rule was used to compute the probability of choosing the gamble option ( $\operatorname{Pr}$ (ChoseGamble) as a function of the difference in subjective value of the gamble ( $\left.\mathrm{EU}_{\mathrm{Gamble}}\right)$ and the safe option $\left(\mathrm{EU}_{\mathrm{Safe}}\right)$. In addition, to account for possible stochasticity in choice, we modeled the decisions of participants as susceptible to an error $(\mu)$ :

$$
\operatorname{Pr}(\text { ChoseGamble })=\frac{1}{1+\exp \left(-\left(\mathrm{EU}_{\text {Gamble }}-\mathrm{EU}_{\text {Safe }}\right) / \mu\right)}
$$

We refitted this function using a grid search procedure to account for local minima in the estimated parameters. The resulting risk and ambiguity attitudes were used as predictors of brain activation in whole-brain regressions. To facilitate interpretation, ambiguity attitude was recoded, such that higher values indicate a more seeking attitude.

\section{MRI Data Acquisition}

We used a 3-T scanner (Philips Achieva TX, Erlangen, Germany) with a standard whole-head coil. Functional scans were acquired during two runs of 246 dynamics each, using T2* EPI. Volumes covered the whole brain (repetition time $=2.2 \mathrm{sec}$, echo time $=30 \mathrm{msec}$, sequential acquisition $=38$ slices, voxel size $=2.75 \times 2.75 \times$ $2.75 \mathrm{~mm}$, field of view $=220 \times 220 \times 114.68 \mathrm{~mm}$ ). We discarded the first two volumes to allow equilibration of T1 saturation effects.

\section{MRI Data Analyses}

\section{Preprocessing}

The data were analyzed using SPM8 (Wellcome Department of Cognitive Neurology, London, United Kingdom). Images were corrected for slice timing acquisition and rigid body motion. Functional volumes were spatially normalized to EPI templates. Translational movement parameters never exceeded $3 \mathrm{~mm}$ ( $<1$ voxel) in any direction for any participant or scan (movement range $=$ $0.00-1.19 \mathrm{~mm}, M=0.058 \mathrm{~mm}, S D=0.020 \mathrm{~mm})$. The normalization algorithm used a 12-parameter affine transform with a nonlinear transformation involving cosine basis function and resampled the volumes to $3-\mathrm{mm}^{3}$ voxels. Templates were based on MNI-305 stereotaxic space. The functional volumes were spatially smoothed using a 6-mm FWHM isotropic Gaussian kernel.

\section{General Linear Model}

To perform statistical analyses on individual participants' data, we used the general linear model in SPM8. The fMRI time series were modeled as a series of two events convolved with the hemodynamic response function. The 
onset of the choice phase was modeled with a duration of choice (1000 msec + response time; see Figure 1). Events were modeled separately for gambling under risk and gambling under ambiguity and for choosing the safe option under risk and choosing the safe option under ambiguity. This resulted in four conditions in the choice phase: gamble risk, gamble ambiguity, safe risk, and safe ambiguity. The onset of the feedback phase (second event) was modeled with zero duration. We modeled gain and no gain after a risky or ambiguous gamble and safe gain after a risky or ambiguous safe choice. This resulted in six conditions in the feedback phase: gain risk, no gain risk, gain ambiguity, no gain ambiguity, safe gain risk, and safe gain ambiguity. Given that outcomes are based on choices, a participant who gambled more frequently viewed more gain and no gain feedback than a participant who chose the safe option $(€ 3)$ more frequently. However, the participants gambled a considerable number of times on average $(M=0.77, S D=$ $0.18)$. This translated into the participants experiencing 36 gains on average $(S D=8.21$, range $=18-46)$ and 35 no gains $(S D=8.33$, range $=17-46$ ) after gambling. Hence, all participants experienced at least 18 gains and 17 no gains, thus leaving a sufficient number of trials for our fMRI analyses on reward outcomes. Furthermore, to check that the prior outcome (gain, no gain) did not influence the neuroimaging results during gambling, we also tested a separate general linear model that included whether the gamble was preceded by a gain or a no gain. Because results remained similar between the two models, we only report the more parsimonious model without reward outcome modeled in the gambling conditions.

Trials on which the participants failed to respond were modeled separately as a covariate of no interest. In addition, six motion parameters were included as nuisance regressors. The least squares parameter estimates of the height of the best-fitting canonical hemodynamic response function for each condition separately were used in pairwise contrasts. These pairwise comparisons resulted in participant-specific contrast images, which were used for second-level group analyses. All second-level group analyses were conducted with family-wise error (FWE) cluster correction $(p<.05$, with a primary voxel-wise threshold of $p<.001$ [uncorrected]; Woo, Krishnan, \& Wager, 2014) or FWE voxel correction $(p<.05)$, indicated where needed. To visualize patterns of activation in clusters identified in the whole-brain regressions, we used the MarsBaR toolbox (Brett, Anton, Valabregue, \& Poline, 2002; marsbar.sourceforge.net). Coordinates of local maxima are reported in MNI space.

Two types of models. In addition to our main model with all various probability trials (i.e., 25\%, 50\%, and $75 \%$ gain probability), we also include in the tables which clusters are present in a model with the ambiguous trials and risky trials with a 50\% probability only. The latter was done because, objectively, the ambiguous trials reflect a 50\% probability (e.g., Levy, 2016; Tymula et al., 2012). For the model with $50 \%$ probability risk trials only, we modeled the other probability risk trials $(25 \%, 75 \%)$ as covariates of no interest.

\section{RESULTS}

\section{Behavioral Results}

Behavioral Task

Results from the model-based estimations showed that participants were predominantly risk and ambiguity averse. That is, most risk attitudes were below $1(M=$ $0.63, S D=0.21$, range $=0.30-1.03)$, and most ambiguity attitudes (after recoding) were below $0(M=-0.30$, $S D=0.37$, range $=-1.0$ to 0.66 ). This general pattern of aversion to risk and ambiguity coincides with prior research (Levy et al., 2010; Huettel et al., 2006), although the range of the attitudes, and inspection of scatterplots, indicated considerable individual differences in aversion to risk and ambiguity (see Figure 2A). Finally, we observed a moderate negative relation between risk and ambiguity attitudes, indicating that more risk seeking was in relation to less ambiguity seeking ( $r=-.22, p=.124$; Figure 2 ). However, this relation was not significant, echoing prior studies that have predominantly found nonsignificant relations between these phenomena (van den Bos \& Hertwig, 2017; Blankenstein et al., 2016; Tymula et al., 2013; Bossaerts, Ghirardato, Guarnaschelli, \& Zame, 2010; Levy et al., 2010).

\section{fMRI Task}

In the fMRI task, when choosing between the safe option and the gamble option (risky or ambiguous), participants gambled significantly less in the ambiguous trials than in the risky trials, as indicated by a paired samples $t$ test $\left(t(49)=-2.35, p=.023 ; M_{\text {Ambig }}=0.75, S D_{\text {Ambig }}=\right.$ $\left.0.25 ; M_{\text {Risk }}=0.82, S D_{\text {Risk }}=0.14\right)$. This effect was more pronounced when comparing the ambiguous trials with the $50 \%$ probability risk trials only $(t(49)=-4.61, p<$ $.001 ; M_{\text {Ambig }}=0.75, S D_{\text {Ambig }}=0.25 ; M_{\text {Risk50 }}=0.90$, $\left.S D_{\text {Risk50 }}=0.18\right)$. Furthermore, when gambling, participants responded slower in the ambiguous trials than in the risky trials in both the model with all trials $(t(49)=$ 4.09, $p<.001 ; M_{\text {Ambig }}=653.73, S D_{\text {Ambig }}=281.95$; $\left.M_{\text {Risk }}=529.83, S D_{\text {Risk }}=163.66\right)$ as well as the model with $50 \%$ probability trials only $(t(49)=3.40, p=.001$; $M_{\text {Ambig }}=653.73, S D_{\text {Ambig }}=281.95 ; M_{\text {Risk50 }}=547.84$, $\left.S D_{\text {Risk50 }}=206.16\right)$. Thus, although participants were encouraged to gamble in both conditions (by offering gambles with relatively high EVs), we still observed that they chose the ambiguous gamble less frequently than the risky gamble and responded slower in the ambiguous compared with risky condition, indicative of a general aversion to ambiguity (Levy et al., 2010; Ellsberg, 1961). 
Figure 2. (A) Relation between risk attitude ( $\alpha, x$ axis) and ambiguity attitude ( $\beta, y$ axis), derived from the behavioral task. Higher values indicate more risk or ambiguity seeking. (B) Proportion gambling ( $y$ axis), across task bins for the risky and ambiguous conditions of the fMRI task. (C) Relation between proportion gambling in the risky condition of the fMRI task ( $x$ axis) and risk attitude derived from the behavioral task ( $\alpha, x$ axis). (D) Relation between proportion gambling in the ambiguous condition of the fMRI task ( $x$ axis) and ambiguity attitude derived from the behavioral task ( $\beta, y$ axis).

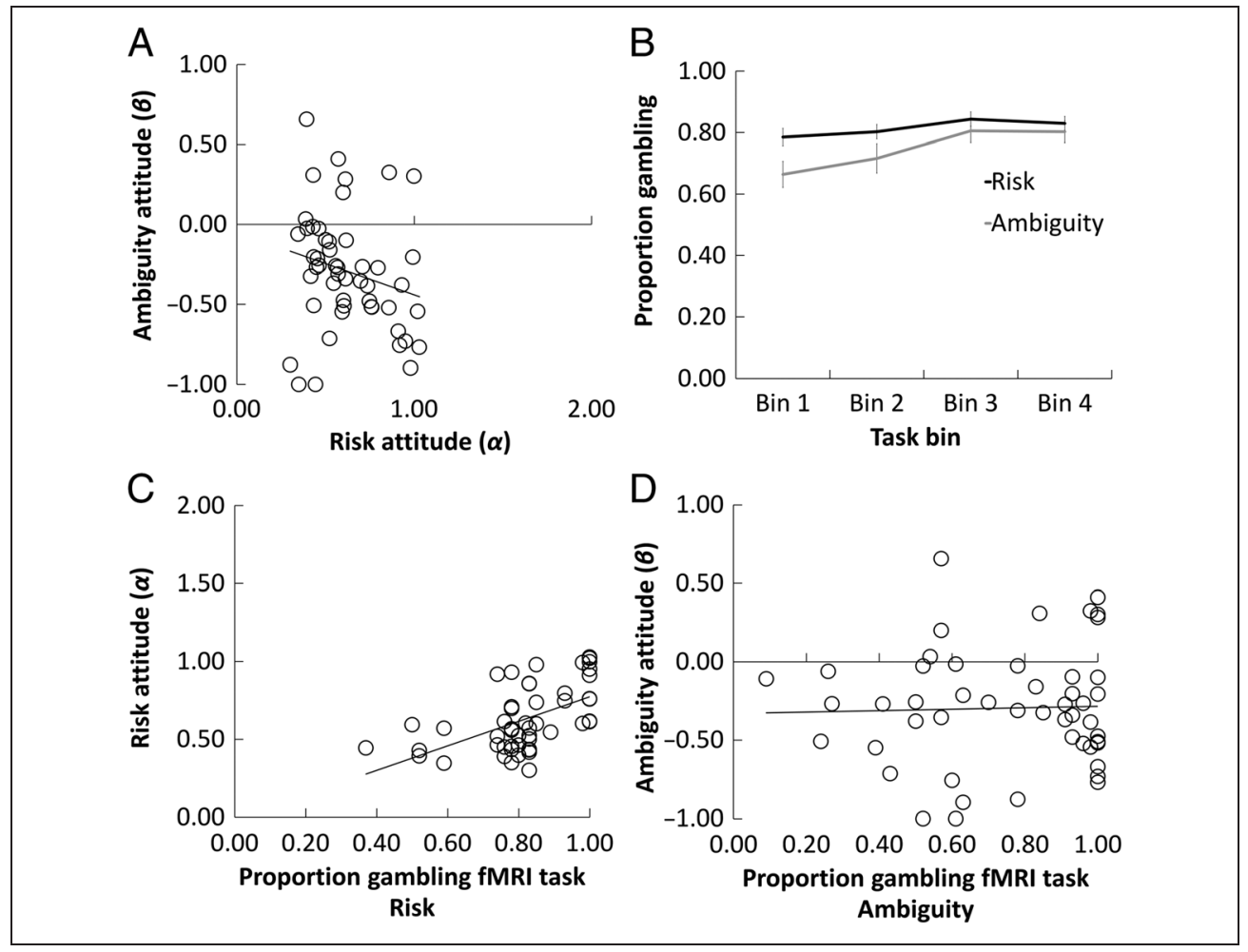

Next, to examine whether feedback influenced behavior in the scanner, we investigated changes in gambling behavior in the fMRI task across time. To this end, we divided behavior across four task bins (with 11 or 12 trials per bin) per condition (risk and ambiguity). A mixed ANOVA showed that, in addition to a main effect of Condition, there was a significant Bin $\times$ Condition interaction $\left(F(3,147)=3.34, p=.021, \eta^{2}=.064\right)$ and a main effect of bin $\left(F(3,147)=9.15, p<.001, \eta^{2}=\right.$ .157). That is, gambling behavior overall increased slightly across time, specifically in the ambiguous condition (see Figure 2B). Similar effects were found when comparing the ambiguous condition with the $50 \%$ probability risk trials only $(\mathrm{Bin} \times$ Condition interaction: $F(3$, $147)=3.27, p=.023, \eta^{2}=.064$; main effect of Bin: $F(3$, $\left.147)=8.57, p<.001, \eta^{2}=.149\right)$.

Finally, we correlated behavior from the fMRI task (proportion gambling in risk and ambiguity) with the model-based estimations of risk and ambiguity attitude (derived from the behavioral task outside the scanner). These analyses showed that risk attitude was positively correlated with proportion gambling in risk $\left(r_{\text {alltrials }}=\right.$ $\left..453, p_{\text {alltrials }}<.001 ; r_{50: 50 \text { trials }}=.317, p_{50: 50 \text { trials }}=.025\right)$ and proportion gambling in ambiguity $(r=.465, p=$ $.001)$. However, ambiguity attitude was not correlated with behavior in the scanner (all $p s>.7$ ). This suggests that relatively more risk-seeking, but not ambiguityseeking, attitudes were associated with a greater general tendency to gamble in the fMRI task (see Figure $2 \mathrm{C}$ and $\mathrm{D}$ ).

\section{fMRI Results}

\section{Whole-brain Contrasts}

Risky and ambiguous gambling. First, to study which brain regions were more strongly activated during gambling under risk versus ambiguity, we calculated the whole-brain contrast Gamble Risk > Gamble Ambiguity and the reversed contrast, based on all probability trials. The first revealed greater activation during gambling under risk in clusters including the right dorsolateral PFC and occipital cortex, extending into bilateral PPC $\left(\mathrm{FWE}_{\mathrm{CC}}\right.$ $p<.05, k>94$; Figure 3A, Table 1). The reversed contrast (Gamble Ambiguity $>$ Gamble Risk) did not result in significant clusters of activation.

Conjunction analysis of risky and ambiguous gambling. To check that regions important for complex decision-making were recruited during our task, we next examined the overlap in brain activation for risky and ambiguous gambling. To this end, we performed a conjunction analysis in which we applied the "Logical AND" strategy, which requires that all comparisons in the conjunction are individually significant (Nichols, Brett, Andersson, Wager, \& Poline, 2005). These particular results are reported at FWE voxel correction $(p<.05)$, because cluster correction resulted in one cluster of activation composed of almost the entire brain, impeding interpretation (Woo et al., 2014). As could be expected, the conjunction analysis revealed widespread overlapping 
Figure 3. (A) Whole-brain contrasts for Gamble Risk > Gamble Ambiguity. Results were FWE cluster corrected $\left(p_{\mathrm{FWE}}<.05, k>94\right)$ with a primary voxel-wise threshold of $p<.001$ (uncorrected). The reversed contrast (Gamble Ambiguity > Gamble Risk) yielded no significant activation. (B) Conjunction of Gamble Risk $>$ Fixation and Gamble Ambiguity $>$ Fixation. Results were FWE voxel-wise corrected $(p<.05)$ and are visualized with $k>10$.

\section{A Gamble Risk > Gamble Ambiguity}

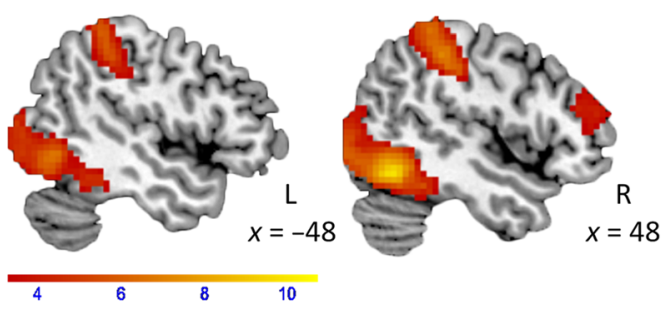

B Conjunction Gamble Risk > Fix and Gamble Ambiguity > Fix
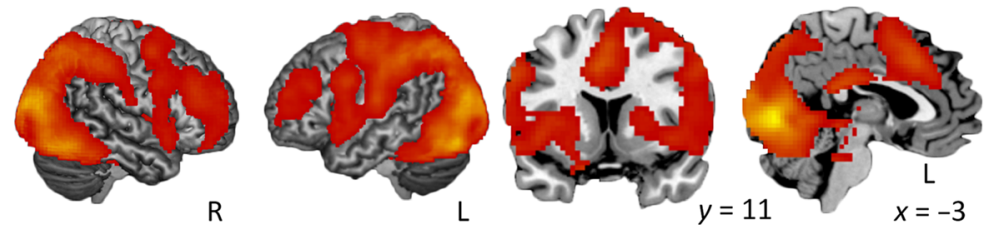

$5 \quad 10 \quad 16 \quad 20$

activation for risky and ambiguous gambling in regions important for risky choice, such as the frontoparietal and parietal regions, including lateral PFC, PPC, ACC, SMA, insula, and putamen (FWE $p<.05$; Table 2, Figure 3B).

Table 1. MNI Coordinates of Local Maxima Activated Clusters for the Contrast Gamble Risk > Gamble Ambiguity

\begin{tabular}{|c|c|c|c|c|c|}
\hline \multirow[b]{2}{*}{ Cluster of Activation } & \multicolumn{3}{|c|}{$\begin{array}{c}M N I \\
\text { Coordinates }\end{array}$} & \multirow[b]{2}{*}{ Significance } & \multirow[b]{2}{*}{ Voxels } \\
\hline & $x$ & $y$ & $z$ & & \\
\hline R inferior temporal gyrus ${ }^{\mathrm{a}}$ & 51 & -58 & -14 & $<.001$ & 7,235 \\
\hline R middle occipital gyrus ${ }^{\mathrm{a}}$ & 36 & -88 & 10 & & \\
\hline L calcarine gyrus $^{\mathrm{a}}$ & 0 & -88 & -2 & & \\
\hline R calcarine gyrus ${ }^{\mathrm{a}}$ & 12 & -100 & -2 & & \\
\hline L superior parietal lobule ${ }^{\mathrm{a}}$ & -27 & -64 & 52 & & \\
\hline L middle occipital gyrus $^{\mathrm{a}}$ & -30 & -85 & 19 & & \\
\hline L superior parietal lobule ${ }^{\mathrm{a}}$ & -15 & -73 & 55 & & \\
\hline L calcarine gyrus $^{\mathrm{a}}$ & -12 & -79 & 10 & & \\
\hline R calcarine gyrus ${ }^{\mathrm{a}}$ & 12 & -103 & 10 & & \\
\hline L superior occipital gyrus $^{\mathrm{a}}$ & -15 & -85 & 7 & & \\
\hline L middle occipital gyrus $^{\mathrm{a}}$ & -39 & -82 & 4 & & \\
\hline $\mathrm{R}$ middle frontal gyrus & 48 & 44 & 16 & $<.001$ & 94 \\
\hline $\mathrm{R}$ inferior frontal gyrus & 45 & 38 & 10 & & \\
\hline R middle frontal gyrus & 45 & 41 & 25 & & \\
\hline
\end{tabular}

Results were FWE cluster corrected ( $p_{\mathrm{FWE}}<.05, k>94$ ) with a primary voxel-wise threshold of $p<.001$ (uncorrected). Results of the reversed contrast (Gamble Ambiguity > Gamble Risk) did not result in significant brain activation. Anatomical labels were acquired with automated anatomical labeling. $\mathrm{L}=$ Left; $\mathrm{R}=$ Right.

${ }^{a}$ Coordinate remained present in the model with $50 \%$ probability trials only.
Whole-brain regressions of risk and ambiguity attitudes. We next tested whether individual differences in risk and ambiguity attitudes (derived from the behavioral task) were related to brain activation during risky and ambiguous gambling using whole-brain regressions. ${ }^{1}$ Given the moderate correlation between risk and ambiguity attitudes $(r=-.22, p=.124)$, we controlled for ambiguity attitude in the regression with risk attitude and for risk attitude in the regression with ambiguity attitude. Specifically, in the regression testing for associations between risk attitude and risky gambling, we entered ambiguity attitude as a covariate of no interest. Likewise, in the regression testing for associations between ambiguity attitude and ambiguous gambling, we entered risk attitude as a covariate of no interest.

First, we observed that a relatively more risk-seeking attitude was associated with increased activation during gambling under risk (Gamble Risk > Fixation) in the medial OFC/ventral ACC and in the left lateral OFC $\left(\mathrm{FWE}_{\mathrm{cc}}\right.$ $p<.05, k>57$; Table 3, Figure 4A). With respect to gambling under ambiguity (Gamble Ambiguity $>$ Fixation), a relatively more ambiguity-seeking attitude was related to increased activation in a cluster of right superior and middle temporal cortex $\left(\mathrm{FWE}_{\mathrm{cc}} p<.05, k>88\right.$; Table 4, Figure $4 \mathrm{~B})$. It should be noted that, when testing brain-behavior associations restricted toward the activation observed in the main contrasts (Gamble Risk > Gamble Ambiguity and vice versa), no associations between risk and ambiguity attitudes and brain activation were observed.

Reward outcome after risky and ambiguous gambling. First, we examined which areas contribute to gain versus no gain irrespective of risk and ambiguity by calculating the contrast Gain > No Gain. These particular results are reported at FWE voxel correction $(p<.05)$ because cluster correction resulted in one cluster of activation 
encompassing almost the entire brain, limiting interpretation (Woo et al., 2014). Here, we observed activation in VS and middle cingulate cortex (FWE $p<.05$; Table 5, Figure 5A).

To compare reward processing (gain versus no gain) after an ambiguous gamble or a risky gamble, we ran a whole-brain repeated-measures ANOVA with Condition (Gain Risk > No Gain Risk, Gain Ambiguity > No Gain

Table 2. MNI Coordinates of Local Maxima Activated for the Conjunction Analysis with Gamble Risk > Fixation and Gamble Ambiguity $>$ Fixation

\begin{tabular}{|c|c|c|c|c|c|}
\hline \multirow[b]{2}{*}{ Area of Activation } & \multicolumn{3}{|c|}{$\begin{array}{c}M N I \\
\text { Coordinates }\end{array}$} & \multirow[b]{2}{*}{$t$} & \multirow[b]{2}{*}{ Voxels } \\
\hline & $x$ & $y$ & $z$ & & \\
\hline $\mathrm{R}$ fusiform gyrus ${ }^{\mathrm{a}}$ & 24 & -76 & -11 & 24.04 & 21,622 \\
\hline R superior occipital gyrus ${ }^{\mathrm{a}}$ & 18 & -94 & 16 & 23.44 & \\
\hline $\mathrm{L}_{\text {lingual gyrus }}^{\mathrm{a}}$ & 3 & -79 & 1 & 23.24 & \\
\hline L middle occipital gyrus ${ }^{\mathrm{a}}$ & -30 & -88 & 19 & 21.80 & \\
\hline L middle occipital gyrus ${ }^{a}$ & -33 & -88 & 13 & 21.26 & \\
\hline 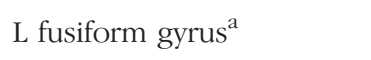 & -27 & -79 & -17 & 20.80 & \\
\hline $\mathrm{R}$ middle occipital gyrus ${ }^{\mathrm{a}}$ & 33 & -88 & 16 & 20.74 & \\
\hline L calcarine gyrus ${ }^{\mathrm{a}}$ & -6 & -85 & -5 & 20.70 & \\
\hline L lingual gyrus ${ }^{\mathrm{a}}$ & -12 & -82 & -8 & 20.66 & \\
\hline L fusiform gyrus ${ }^{\mathrm{a}}$ & -24 & -70 & -14 & 20.26 & \\
\hline $\mathrm{L}_{\text {cerebellum }}{ }^{\mathrm{a}}$ & -18 & -82 & -17 & 20.24 & \\
\hline L lingual gyrus ${ }^{\mathrm{a}}$ & -18 & -79 & -11 & 20.09 & \\
\hline L middle occipital gyrus ${ }^{\mathrm{a}}$ & -12 & -100 & 1 & 19.33 & \\
\hline R calcarine gyrus ${ }^{\mathrm{a}}$ & 12 & -94 & 4 & 19.02 & \\
\hline L calcarine gyrus ${ }^{\mathrm{a}}$ & -12 & -79 & 4 & 18.84 & \\
\hline L middle occipital gyrus ${ }^{a}$ & -18 & -97 & 10 & 18.37 & \\
\hline L middle frontal gyrus ${ }^{\mathrm{a}}$ & -45 & 38 & 31 & 9.72 & 383 \\
\hline L superior frontal gyrus ${ }^{\mathrm{a}}$ & -30 & 47 & 40 & 6.48 & \\
\hline L superior orbital gyrus ${ }^{\mathrm{a}}$ & -30 & 62 & -2 & 5.65 & \\
\hline $\mathrm{R}$ middle frontal gyrus ${ }^{\mathrm{b}}$ & 45 & 44 & 28 & 9.93 & 461 \\
\hline $\mathrm{R}$ middle orbital gyrus ${ }^{\mathrm{b}}$ & 45 & 47 & -17 & 6.12 & \\
\hline R superior frontal gyrus ${ }^{b}$ & 27 & 53 & 37 & 5.99 & \\
\hline $\mathrm{R}$ middle frontal gyrus ${ }^{\mathrm{b}}$ & 42 & 59 & 4 & 5.94 & \\
\hline $\mathrm{R}$ middle orbital gyrus ${ }^{\mathrm{b}}$ & 45 & 53 & -5 & 5.86 & \\
\hline
\end{tabular}

Results were FWE voxel-wise corrected $(p<.05)$. Only areas of activation larger than 10 contiguous voxels are reported. Anatomical labels were acquired with automated anatomical labeling.

${ }^{a}$ Local maximum remained present in the model with $50 \%$ probability risk trials only.

${ }^{\mathrm{b}}$ Local maximum was additionally present in the model with $50 \%$ probability risk trials only.
Table 3. MNI Coordinates of Local Maxima Activated Clusters for the Contrast Gamble Risk > Fixation with Risk Attitude as a Positive Regressor

\begin{tabular}{|c|c|c|c|c|c|}
\hline \multirow[b]{2}{*}{ Cluster of Activation } & \multicolumn{3}{|c|}{$\begin{array}{c}\text { MNI } \\
\text { Coordinates }\end{array}$} & \multirow[b]{2}{*}{ Significance } & \multirow[b]{2}{*}{ Voxels } \\
\hline & $x$ & $y$ & $z$ & & \\
\hline L middle orbital gyrus & -27 & 41 & -11 & .033 & 57 \\
\hline L middle orbital gyrus & -33 & 47 & -14 & & \\
\hline L middle orbital gyrus & -21 & 32 & -20 & & \\
\hline $\begin{array}{l}\text { R olfactory cortex- } \\
\text { caudate nucleus }\end{array}$ & 3 & 17 & -11 & .013 & 71 \\
\hline L rectal gyrus & -9 & 17 & -14 & & \\
\hline $\begin{array}{l}\text { L inferior frontal gyrus } \\
\text { (pars orbitalis) }\end{array}$ & -18 & 23 & -17 & & \\
\hline L rectal gyrus & -12 & 26 & -14 & & \\
\hline R olfactory cortex & 6 & 23 & -5 & & \\
\hline L olfactory cortex & -12 & 14 & -17 & & \\
\hline
\end{tabular}

Results were FWE cluster corrected ( $p_{\mathrm{FWE}}<.05, k>57$ ) with a primary voxel-wise threshold of $p<.001$ (uncorrected). Anatomical labels were acquired with automated anatomical labeling.

Ambiguity) as the within-participant factor. Results of the ANOVA showed that reward processing after an ambiguous gamble, compared with a risky gamble, revealed increased activation in the dorsomedial PFC (MNI coordinates: $x=12, y=29, z=52 ; \mathrm{FWE}_{\mathrm{cc}} p<.05, k=52$; Figure 5B). The reversed effect ([Gain Risk $>$ No Gain Risk] > [Gain Ambiguity > No Gain Ambiguity]) was not associated with significant brain activation.

Finally, no associations between risk and ambiguity attitudes and brain activation during reward processing were observed.

\section{DISCUSSION}

This study aimed to elucidate specific neural systems underlying risk (known probabilities) and ambiguity (unknown probabilities) processing at the individual level. Specifically, we investigated the association between individuals' risk and ambiguity attitudes and brain activation during gambling, tested the role of risk and ambiguity within a choice at hand on subsequent neural reward processing, and explored associations between risk and ambiguity attitudes and reward processing after a risky and ambiguous gamble. To these ends, we combined an fMRI gambling paradigm with separately established model-based estimations of risk and ambiguity attitudes. Results showed that there was variability between individuals in risk and ambiguity attitudes and that these attitudes were only moderately and nonsignificantly correlated. This allowed the investigation of risk and ambiguity attitudes as individual predictors of the neural 
Figure 4. (A) The positive effect of risk attitude $(\alpha)$ on risky gambling (Gamble Risk > Fixation), controlled for panel shows the positive relation between risk attitude ( $x$ axis) on brain activation ( $y$ axis) in the medial OFC. Results were FWE cluster corrected $\left(p_{\mathrm{FWE}}<.05\right.$, $k>57$ ) with a primary voxelwise threshold of $p<.001$ (uncorrected). (B) The positive effect of ambiguity attitude $(\beta)$ on ambiguous gambling (Gamble Ambiguity > Fixation), controlled for risk attitude. The right panel shows the positive relation between ambiguity attitude ( $x$ axis) and brain activation ( $y$ axis) in the right superior temporal gyrus. Results were FWE cluster corrected $\left(p_{\text {FWE }}<.05, k>88\right)$ with a primary voxel-wise threshold of $p<.001$ (uncorrected). The graphs are for illustrative purposes only. No statistical analyses were performed on the ROIs. ambiguity attitude. The right

\section{A Positive association with risk attitude}
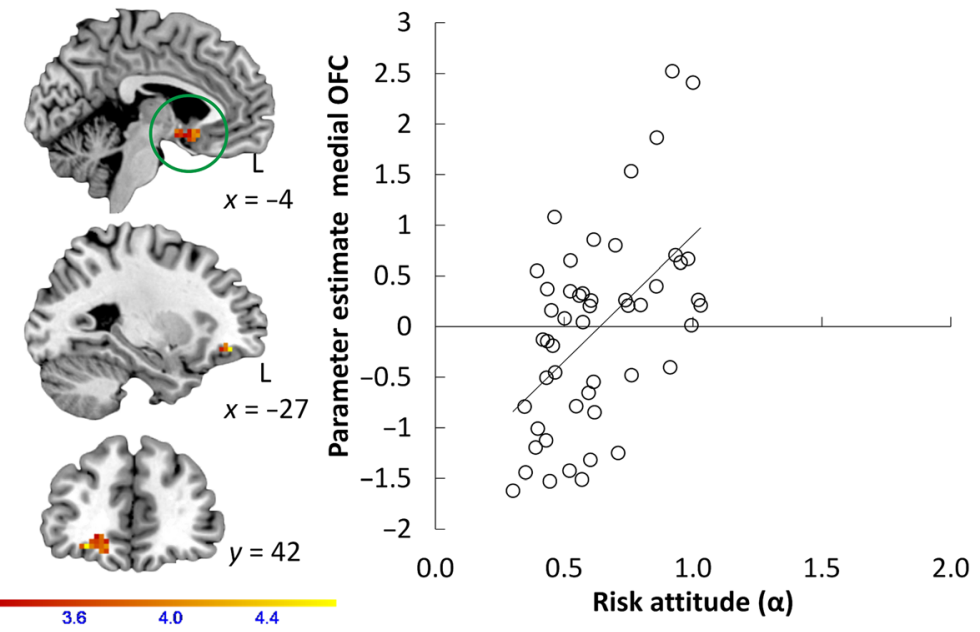

B Positive association with ambiguity attitude
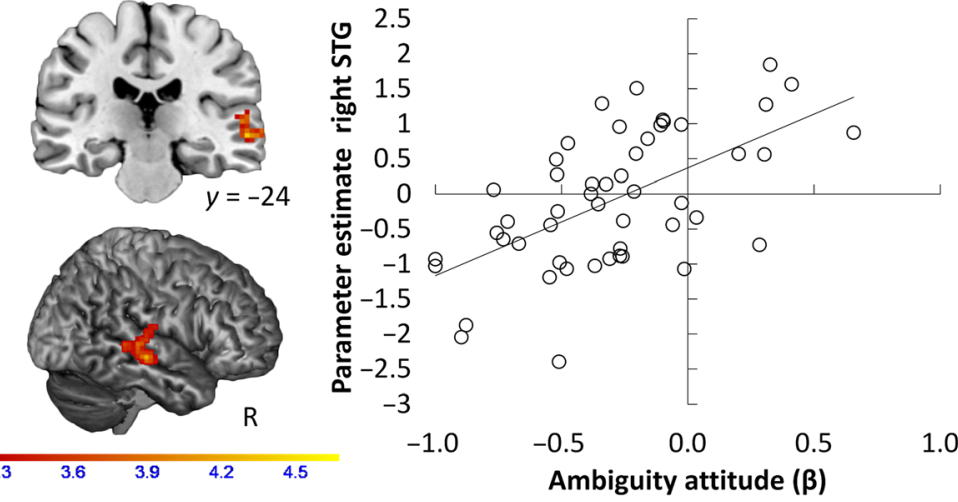

processes underlying risky and ambiguous decisionmaking. The fMRI analyses resulted in a number of main findings. First, despite pronounced neural overlap between risky and ambiguous gambling in a network previously associated with risk taking and decision-making (e.g., see

Table 4. MNI Coordinates of Local Maxima Activated Clusters for the Contrast Gamble Ambiguity > Fixation with Ambiguity Attitude as a Positive Regressor, for the Model Including All Risk Trials

\begin{tabular}{|c|c|c|c|c|c|}
\hline \multirow[b]{2}{*}{ Cluster of Activation } & \multicolumn{3}{|c|}{$\begin{array}{c}M N I \\
\text { Coordinates }\end{array}$} & \multirow[b]{2}{*}{ Significance } & \multirow[b]{2}{*}{ Voxels } \\
\hline & $x$ & $y$ & $z$ & & \\
\hline $\mathrm{R}$ superior temporal gyrus & 63 & -22 & -5 & .008 & 88 \\
\hline $\mathrm{R}$ middle temporal gyrus & 57 & -28 & 1 & & \\
\hline $\mathrm{R}$ middle temporal gyrus & 57 & -34 & 1 & & \\
\hline $\mathrm{R}$ rolandic operculum & 57 & -16 & 16 & & \\
\hline
\end{tabular}

Results were FWE cluster corrected ( $p_{\mathrm{FWE}}<.05, k>88$ ) with a primary voxel-wise threshold of $p<.001$ (uncorrected). Anatomical labels were acquired with automated anatomical labeling.
Levy, 2016; Platt \& Huettel, 2008; Eshel, Nelson, Blair, Pine, \& Ernst, 2007; Huettel et al., 2005; Knutson et al., 2005; Kuhnen \& Knutson, 2005; Ernst et al., 2004), individual differences in risk and ambiguity attitudes showed different neural substrates during risky and ambiguous gambling, respectively. That is, we observed that a relatively more risk-seeking attitude was associated with increased activation in medial and lateral OFC during risky gambling and that a relatively more ambiguity-seeking attitude was related to increased activation in superior and middle temporal gyrus during ambiguous gambling. Second, processing rewards relative to no rewards resulted in robust activity in the VS, irrespective of a previous risky or ambiguous gamble. However, processing rewards compared with no rewards after an ambiguous gamble, compared with after a risky gamble, resulted in increased dorsomedial PFC activation. Finally, risk and ambiguity attitudes were not correlated with any neural activity during reward processing. The discussion is organized alongside these main findings.

The main question addressed in this study was whether risk and ambiguity processing relied on different neural substrates. Both risky and ambiguous gambling were 
Table 5. MNI Coordinates of Local Maxima Activated for the Contrast Gain > No Gain, Irrespective of Risk and Ambiguity

\begin{tabular}{|c|c|c|c|c|c|}
\hline \multirow[b]{2}{*}{ Area of Activation } & \multicolumn{3}{|c|}{$\begin{array}{c}M N I \\
\text { Coordinates }\end{array}$} & \multirow[b]{2}{*}{$t$} & \multirow[b]{2}{*}{ Voxels } \\
\hline & $x$ & $y$ & $z$ & & \\
\hline L putamen ${ }^{\mathrm{a}}$ & -15 & 8 & -14 & 8.93 & 110 \\
\hline R putamen ${ }^{\mathrm{a}}$ & 12 & 5 & -17 & 7.57 & 76 \\
\hline $\mathrm{R}$ caudate nucleus ${ }^{\mathrm{a}}$ & 9 & 11 & -11 & 7.30 & \\
\hline L superior frontal gyrus ${ }^{\mathrm{a}}$ & -18 & 23 & 58 & 7.01 & 55 \\
\hline L precentral gyrus & -24 & -23 & 58 & 6.96 & \\
\hline $\mathrm{L}_{\text {middle frontal gyrus }}{ }^{\mathrm{a}}$ & -21 & 26 & 55 & 6.68 & \\
\hline L superior frontal gyrus & -15 & 32 & 46 & 5.60 & \\
\hline R middle occipital gyrus ${ }^{\mathrm{a}}$ & 51 & -70 & 28 & 6.67 & 17 \\
\hline L inferior parietal lobe & -48 & -40 & 49 & 6.45 & 21 \\
\hline $\mathrm{L} / \mathrm{R}$ middle cingulate cortex & 0 & -40 & 43 & 6.24 & 25 \\
\hline $\mathrm{R}$ middle cingulate cortex & 3 & -34 & 40 & 5.74 & \\
\hline $\mathrm{R}$ middle cingulate cortex & 3 & -40 & 34 & 5.44 & \\
\hline R superior frontal gyrus & 24 & 26 & 49 & 6.01 & 10 \\
\hline L rectal gyrus ${ }^{b}$ & -3 & 41 & -17 & 6.38 & 15 \\
\hline $\mathrm{R} / \mathrm{L}$ middle orbital gyrus ${ }^{\mathrm{b}}$ & 0 & 44 & -14 & 5.96 & \\
\hline L superior frontal gyrus ${ }^{\mathrm{b}}$ & -21 & 41 & 40 & 6.08 & 17 \\
\hline
\end{tabular}

Results were FWE voxel-wise corrected $(p<.05)$. Only areas of activation larger than 10 contiguous voxels are reported. Anatomical labels were acquired with automated anatomical labeling.

${ }^{a}$ Local maximum remained present in the model with only $50 \%$ gain probability risk trials.

${ }^{\mathrm{b}}$ Local maximum was additionally present in the model with $50 \%$ probability risk trials only.

associated with robust activity in ACC, PPC, lateral PFC, striatum (putamen), and insula, regions commonly observed in various risk-taking paradigms (Levy, 2016; Platt \& Huettel, 2008; Eshel et al., 2007; Huettel et al., 2005; Knutson et al., 2005; Kuhnen \& Knutson, 2005; Ernst et al., 2004). The main comparisons between risk and ambiguity revealed little differences between risky and ambiguous gambling. This was unexpected given that prior studies did observe differences between these conditions, although not all in consistent or overlapping directions (Levy et al., 2010; Huettel et al., 2006; Hsu et al., 2005). However, neural differences between risky and ambiguous gambling were observed when we related neural responses to individuals' attitudes toward risk and ambiguity. The use of model-based estimations has the advantage that they reflect a sensitive measure of an individual's preference for risk and ambiguity and are derived from an integrative choice model that simultaneously estimates risk and ambiguity attitude (Blankenstein et al., 2016; Tymula et al., 2013).
From these analyses, we observed that a relatively more risk-seeking attitude was related to increased activation in the medial and lateral OFC during risky gambling. Particularly, the activation observed in the medial OFC coincides with prior studies that also used modelbased estimations of risk attitudes and observed that relatively less risk aversion (Tobler et al., 2007) or more risk seeking (Engelmann \& Tamir, 2009) was associated with increased activation in the medial OFC. Possibly, this activation reflects the influence of individual differences in risk attitude in a region commonly associated with the coding of EV or subjective value (Levy \& Glimcher, 2012; Tobler et al., 2007) and may suggest the enhanced recruitment of this area in individuals who exhibit relatively more risk-seeking behavior. With respect to ambiguity attitude, we observed increased activation in superior and middle temporal gyrus with relatively more ambiguity-seeking attitudes. Together, these analyses suggest that, despite the large overlap in the general network that was engaged when making risky and ambiguous gambles, the way these regions are engaged depends on individual differences in attitudes toward risk and ambiguity.

Not all findings that were reported in previous studies could be confirmed in the current study. Contrary to prior studies, we did not find a relation between individuals' risk attitudes and activation of frontoparietal regions (e.g., inferior frontal gyrus, lateral PFC, PPC; Gilaie-Dotan et al., 2014; Christopoulos et al., 2009; Fecteau et al., 2007; Huettel et al., 2006). Unexpectedly, we also did not observe a relation between ambiguity attitude and lateral PFC or OFC activation (Huettel et al., 2006; Hsu et al., 2005). In part, this could be attributed to the use of model-free measures of risk behavior in some of these studies (e.g., behavior on the Balloon Analogue Risk Task; Fecteau et al., 2007; Knoch et al., 2006), which may be a different measure of someone's risk and ambiguity preferences. Furthermore, some of these abovementioned studies used TMS/direct current stimulation or structural brain measures (i.e., gray matter volume), which may provide different but complementary information on risk and ambiguity processes (Gilaie-Dotan et al., 2014; Fecteau et al., 2007; Knoch et al., 2006). In addition, some of these studies offered choices between two gambles (with varying levels of risk) instead of offering a choice between a gamble and a safe option, iteratively manipulated the value of the safe option based on participants' prior choices, or derived risk and ambiguity attitudes from different choice paradigms (Christopoulos et al., 2009; Huettel et al., 2006; Hsu et al., 2005). Finally, although our paradigm included variation in risk, it only included one level of ambiguity (see for a parametric approach, Levy et al., 2010), leading to small(er) variations in subjective value of the latter. Together, the abovementioned elaborate manipulations are useful additions for future research. Alternatively, in the current study, we examined neural correlates of each attitude while accounting for the other attitude. Thus, although our find- 
Figure 5. (A) Whole-brain contrast for Gain > No Gain. Results were FWE voxel-wise corrected at $p<.05$ and visualized here with $k>10$. (B) Results of the whole-brain repeated-measures ANOVA showing the contrast [Gain Ambiguity $>$ No Gain Ambiguity $]>$ [Gain Risk $>\mathrm{No}$ Gain Risk]. Results were FWE cluster corrected $(p<.05, k>$ 52 ), with a primary voxel-wise threshold of $p<.001$ (uncorrected). The right panel shows the parameter estimate of the dorsomedial PFC, plotted for ambiguity and risk (for visual illustration only, no analyses were performed on the ROI). (C) Neurosynth meta-analysis of fMRI activations associated with the search term "uncertainty" (reverse inference, false discovery rate corrected $=0.01$ ) based on 98 studies.

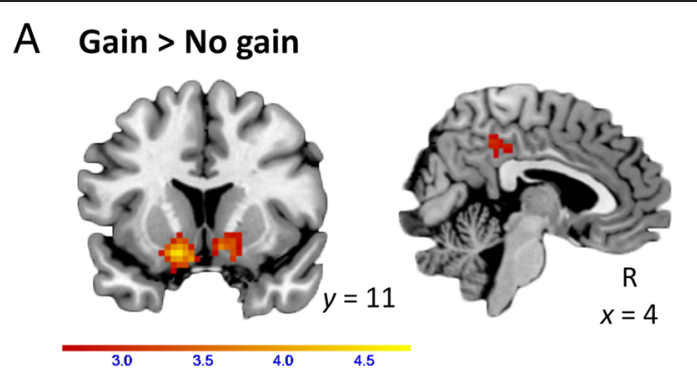

B [Ambiguity Gain $>$ No gain] $>$ [Risk Gain $>$ No gain]
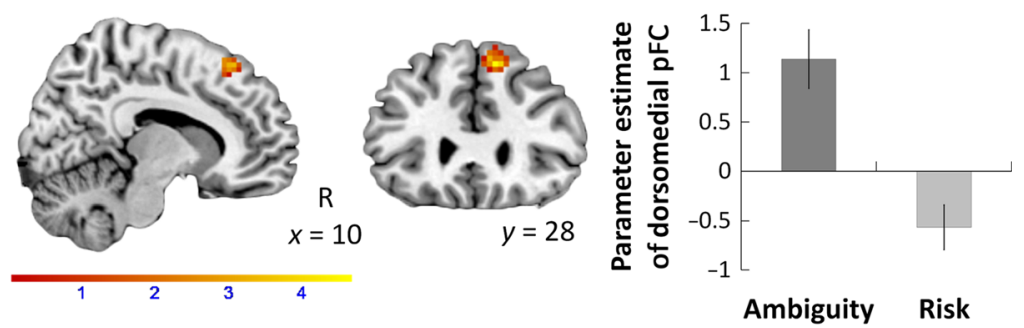

C Neurosynth term "uncertainty"

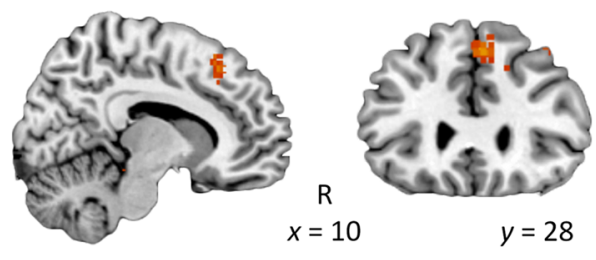

ings warrant replication, they may be more specific for an individual's risk and ambiguity attitudes.

A second question that we aimed to address was whether risk and ambiguity within a choice at hand influence the subsequent neural reward processing (gain vs. no gain). We observed that reward processing irrespective of a prior risky or ambiguous gamble, and independent of risk or ambiguity attitude, resulted in a robust striatal response, replicating many prior studies (e.g., see Braams, Peters, Peper, Güroğlu, \& Crone, 2014; Delgado, 2007; Knutson et al., 2005; Kuhnen \& Knutson, 2005). This suggests that the striatum has a general reward signaling function and is not dependent on the nature of the gamble (i.e., known or unknown probabilities). A second finding was that reward processing after an ambiguous gamble compared with a risky gamble revealed increased activation in the dorsomedial PFC. This activity coincides with research on uncertainty processing in general. That is to say, a reversed inference search with the term "uncertainty" in the Neurosynth database (on online meta-analysis database: www. neurosynth.org; Yarkoni, Poldrack, Nichols, Van Essen, \& Wager, 2012) shows that the dorsomedial PFC is robustly documented in 98 studies on various forms of uncertainty processing (see Figure 5C), for instance, with respect to risky decision-making (e.g., prediction uncertainty; Volz, Schubotz, \& von Cramon, 2003, 2004), and even with respect to self-reported intolerance to uncertainty about possible future aversive events (Schienle, Köchel, Ebner,
Reishofer, \& Schäfer, 2010). The fact that we observed this activation in our study during reward processing after an ambiguous gamble fits well with the interpretation that this region represents a common neural substrate for uncertainty coding.

Finally, we explored whether individuals who differ in risk or ambiguity attitude also process outcomes of gambles differently, which has not yet been examined in prior research. No associations between risk or ambiguity attitude and brain activation during reward processing were observed. Possibly, individual differences in risk and ambiguity preferences are only reflected by the neural mechanisms underlying choices and not the subsequent processing of outcomes. However, future research should further establish this finding.

A number of limitations need to be taken into consideration. First, although we observed different neural mechanisms underlying individuals' risk and ambiguity attitudes during risky and ambiguous gambling, respectively, we stress that we did not observe a dissociation. That is, when investigating whether risk and ambiguity attitudes were associated with activation observed in the main contrasts (such as Risk > Ambiguity), no relations were found with risk or ambiguity attitudes. However, individual differences in risk and ambiguity attitudes may not necessarily be reflected in the activation that is homogeneous for the group as a whole. Rather, individual differences in risk and ambiguity attitudes may be reflected in neural systems that 
show heterogeneity across participants (e.g., see van Duijvenvoorde, Figner, et al., 2016; Gabrieli, Ghosh, \& Whitfield-Gabrieli, 2015). Nevertheless, future studies need to replicate these findings using larger sample sizes. Second, we used a task to define individuals' risk and ambiguity attitudes that differed on several aspects from the functional imaging task. That is, given our interest of neural processes underlying risky and ambiguous gambling, we manipulated the fMRI paradigm such that participants were more likely to gamble than to choose the safe option. That is, the EV of the gamble option was considerably higher (i.e., between $€ 7.75$ and $€ 25.5$ ) than the safe option (€3). In addition, in the behavioral task, participants did not observe direct outcomes and could not win any money, whereas in the fMRI task, participants were told that they won the amount of three randomly chosen trials. Thus, we cannot know for certain whether participants considered the decisions in the behavioral task equally important as those in the fMRI task and whether participants believed that they were getting paid according to what was explained in the instructions in the fMRI task. Given that different behavioral and even brain patterns may emerge when using real versus hypothetical gambles (e.g., see Camerer \& Mobbs, 2016), it is important to ensure that participants consider the gambles in both tasks equally important and that the payouts are believable. In future research, this could be achieved by informing participants that three trials will be randomly played for real for each task and preferably by letting the participants exert control over these randomly chosen trials (Levy et al., 2010). Furthermore, the behavioral task was always administered after the fMRI session, which may have affected the model-based estimations of risk and ambiguity attitudes. Thus, when using a similar setup, future studies may benefit from counterbalancing the order of tasks to eliminate its effect. In addition, whereas risk attitude was positively related to gambling behavior in the fMRI task, we did not observe this between-task correlation for ambiguity attitude. Possibly, this is because we included only one level of ambiguity in the fMRI task, thereby limiting the variation in choice behavior. Whether the cognitive processes underlying both tasks are truly comparable is therefore difficult to establish, and thus, the brain-behavior associations observed in the current study need to be replicated in future research preferably with an fMRI task that allows the estimation of risk and ambiguity attitudes from behavior in the scanner, while simultaneously enabling the investigation of reward processing after a risky and ambiguous gamble. Finally, the current study only focused on gambling in the gain frame. Future studies may benefit from also including a loss frame, for example, to study gain versus loss or loss versus no loss, in risky and ambiguous conditions.

In conclusion, the current study aimed to provide a clear view of the neural substrates of risk and ambiguity processing at the individual level, the association between risk and ambiguity within a choice and subsequent reward processing, and associations between risk and ambiguity attitudes and reward processing. We show that, despite the large overlap in the general network that was engaged during risky and ambiguous gambling, brain activation during gambling depends on individual differences in attitudes toward risk and ambiguity. Especially, the neural correlates of risk attitudes during gambling under risk including medial OFC are consistent with a large body of literature suggesting that this valuation network drives risk taking (Levy \& Glimcher, 2012; Platt \& Huettel, 2008; Delgado, 2007; Knutson et al., 2005; Kuhnen \& Knutson, 2005). The fact that we did not observe this pattern of brain activation with ambiguity attitude suggests that different neural correlates were associated with attitudes toward risk and toward ambiguity. Moreover, these findings highlight the importance of taking individual differences into account that may be masked by group effects. In addition, we found evidence that reward processing after an ambiguous, compared with risky, gamble is related to a heightened dorsomedial PFC response, indicative of a general signal of uncertainty coding. These insights may be applied to future research investigating individual differences in problematic decision-making behavior, such as pathological gambling and adolescent risk taking, which is associated with a heightened sensitivity toward rewards (van Duijvenvoorde, Peters, Braams, \& Crone, 2016; Braams, van Duijvenvoorde, Peper, \& Crone, 2015).

\section{Acknowledgments}

This work was supported by an innovative ideas grant of the European Research Council (ERC-2010-StG-263234 awarded to E. A. C.).

Reprint requests should be sent to Neeltje E. Blankenstein, Department of Developmental and Educational Psychology, Leiden University, Wassenaarseweg 522333 AK Leiden, The Netherlands, or via e-mail: n.e.blankenstein@fsw.leidenuniv.nl.

\section{Note}

1. A different approach to test for effects of individual differences in dealing with risk and ambiguity would be to probe for regions that correlate with subjective value modeled as a parametric modulator separately during risky decision-making and during ambiguous decision-making (cf. Levy et al., 2010). Although our paradigm was not optimized for parametric analyses (i.e., the current fMRI task includes little variation in risk and none in ambiguity) and comparing subjective value under risk versus ambiguity was not the goal of the current study, these analyses were performed for possible future meta-analytical purposes only. In brief, we only observed a positive parametric effect of subjective value under risk in the right parietal cortex, namely, in the right supramarginal gyrus (MNI coordinates: [63, $-31,28]$, FWEcc $p=.026, k=54$ ). Further details and results are available upon request by contacting the first author).

\section{REFERENCES}

Bartra, O., McGuire, J. T., \& Kable, J. W. (2013). The valuation system: A coordinate-based meta-analysis of BOLD fMRI 
experiments examining neural correlates of subjective value. Neuroimage, 76, 412-427.

Blankenstein, N. E., Crone, E. A., van den Bos, W., \& van Duijvenvoorde, A. C. K. (2016). Dealing with uncertainty: Testing risk- and ambiguity-attitude across adolescence. Developmental Neuropsychology, 1, 77-92.

Bossaerts, P., Ghirardato, P., Guarnaschelli, S., \& Zame, W. (2010). Ambiguity in asset Markets: Theory and experiment. Review of Financial Studies, 23, 1325-1359.

Braams, B. R., Peters, S., Peper, J. S., Güroğlu, B., \& Crone, E. A. (2014). Gambling for self, friends, and antagonists: Differential contributions of affective and social brain regions on adolescent reward processing. Neuroimage, 100, 281-289.

Braams, B. R., van Duijvenvoorde, A. C. K., Peper, J. S., \& Crone, E. A. (2015). Longitudinal changes in adolescent risk-taking: A comprehensive study of neural responses to rewards, pubertal development, and risk-taking behavior. Journal of Neuroscience, 35, 7226-7238.

Brett, M., Anton, J., Valabregue, R., \& Poline, J. (2002). Region of interest analysis using an SMP toolbox. Paper presented at 8th International Conference on Functional Mapping of the Human Brain.

Camerer, C., \& Mobbs, D. (2016). Differences in behavior and brain activity during hypothetical and real choices. Trends in Cognitive Sciences, 21, 46-56.

Christopoulos, G. I., Tobler, P. N., Bossaerts, P., Dolan, R. J., \& Schultz, W. (2009). Neural correlates of value, risk, and risk aversion contributing to decision making under risk. Journal of Neuroscience, 29, 12574-12583.

Dale, A. M. (1999). Optimal experimental design for eventrelated fMRI. Human Brain Mapping, 8, 109-114.

Delgado, M. R. (2007). Reward related responses in the human striatum. Annals of the New York Academy of Sciences, 1104, $70-88$.

Delgado, M. R., Nystrom, L. E., Fissell, C., Noll, D., \& Fiez, J. A. (2000). Tracking the hemodynamic responses to reward and punishment in the striatum. Journal of Neurophysiology, 84, 3072-3077.

Ellsberg, D. (1961). Risk, ambiguity, and the Savage axioms. Quarterly Journal of Economics, 75, 643-669.

Engelmann, J. B., \& Tamir, D. (2009). Individual differences in risk preference predict neural responses during financial decision-making. Brain Research, 1290, 28-51.

Ernst, M., Nelson, E. E., McClure, E. B., Monk, C. S., Munson, S., Eshel, N., et al. (2004). Choice selection and reward anticipation: An fMRI study. Neuropsychologia, 42, $1585-1597$.

Eshel, N., Nelson, E. E., Blair, R. J., Pine, D. S., \& Ernst, M. (2007). Neural substrates of choice selection in adults and adolescents: Development of the ventrolateral prefrontal and anterior cingulate cortices. Neuropsychologia, 45, 1270-1279.

Fecteau, S., Pascual-Leone, A., Zald, D. H., Liguori, P., Theoret, H., Boggio, P. S., et al. (2007). Activation of prefrontal cortex by transcranial direct current stimulation reduces appetite for risk during ambiguous decision making. Journal of Neuroscience, 27, 6212-6218.

Gabrieli, J. D., Ghosh, S. S., \& Whitfield-Gabrieli, S. (2015). Prediction as a humanitarian and pragmatic contribution from human cognitive neuroscience. Neuron, 85, 11-26.

Gilaie-Dotan, S., Tymula, A., Cooper, N., Kable, J. W., Glimcher, P. W., \& Levy, I. (2014). Neuroanatomy predicts individual risk attitudes. Journal of Neuroscience, 34, 12394-12401.

Gilboa, I., \& Schmeidler, D. (1989). Maxmin expected utility with non-unique prior. Journal of Mathematical Economics, 18, 141-153.
Hsu, M., Bhatt, M., Adolphs, R., Tranel, D., \& Camerer, C. F. (2005). Neural systems responding to degrees of uncertainty in human decision-making. Science, 310, 1680-1683.

Huettel, S. A., Song, A. W., \& McCarthy, G. (2005). Decisions under uncertainty: Probabilistic context influences activation of prefrontal and parietal cortices. Journal of Neuroscience, 25, 3304-3311.

Huettel, S. A., Stowe, C. J., Gordon, E. M., Warner, B. T., \& Platt, M. L. (2006). Neural signatures of economic preferences for risk and ambiguity. Neuron, 49, 765-775.

Knight, F. H. (1921). Risk, uncertainty and profit. New York: Hart, Schaffner and Marx.

Knoch, D., Gianotti, L. R. R., Pascual-Leone, A., Treyer, V., Regard, M., Hohmann, M., et al. (2006). Disruption of right prefrontal cortex by low-frequency repetitive transcranial magnetic stimulation induces risk-taking behavior. Journal of Neuroscience, 26, 6469-6472.

Knutson, B., Taylor, J., Kaufman, M., Peterson, R., \& Glover, G. (2005). Distributed neural representation of expected value. Journal of Neuroscience, 25, 4806-4812.

Kuhnen, C. M., \& Knutson, B. (2005). The neural basis of financial risk taking. Neuron, 47, 763-770.

Levy, D. J., \& Glimcher, P. W. (2012). The root of all value: A neural common currency for choice. Current Opinion in Neurobiology, 22, 1027-1038.

Levy, I. (2016). Neuroanatomical substrates for risk behavior. Neuroscientist, 23, 275-286.

Levy, I., Snell, J., Nelson, A. J., Rustichini, A., \& Glimcher, P. W. (2010). Neural representation of subjective value under risk and ambiguity. Journal of Neurophysiology, 103, 1036-1047.

Mohr, P. N., Biele, G., \& Heekeren, H. R. (2010). Neural processing of risk. Journal of Neuroscience, 30, 6613-6619.

Nichols, T., Brett, M., Andersson, J., Wager, T., \& Poline, J.-B. (2005). Valid conjunction inference with the minimum statistic. Neuroimage, 25, 653-660.

O’Doherty, J., Kringelbach, M. L., Rolls, E. T., Hornak, J., \& Andrews, C. (2001). Abstract reward and punishment representations in the human orbitofrontal cortex. Nature Neuroscience, 4, 95-102.

O'Doherty, J. P. (2004). Reward representations and rewardrelated learning in the human brain: Insights from neuroimaging. Current Opinion in Neurobiology, 14, 769-776.

Platt, M. L., \& Huettel, S. A. (2008). Risky business: The neuroeconomics of decision making under uncertainty. Nature Neuroscience, 11, 398-403.

Pushkarskaya, H., Smithson, M., Joseph, J. E., Corbly, C., \& Levy, I. (2015). Neural correlates of decision-making under ambiguity and conflict. Frontiers in Behavioral Neuroscience, 9, 325

R Core Team. (2015). R: A language and environment for statistical computing. Vienna: R Foundation for Statistical Computing. Retrieved from www.R-project.org/.

Rao, H., Korczykowski, M., Pluta, J., Hoang, A., \& Detre, J. A. (2008). Neural correlates of voluntary and involuntary risk taking in the human brain: An fMRI study of the Balloon Analog Risk Task (BART). Neuroimage, 42, 902-910.

Schienle, A., Köchel, A., Ebner, F., Reishofer, G., \& Schäfer, A. (2010). Neural correlates of intolerance of uncertainty. Neuroscience Letters, 479, 272-276.

Tobler, P. N., O’Doherty, J. P., Dolan, R. J., \& Schultz, W. (2007). Reward value coding distinct from risk attitude-related uncertainty coding in human reward systems. Journal of Neurophysiology, 97, 1621-1632.

Tricomi, E. M., Delgado, M. R., \& Fiez, J. A. (2004). Modulation of caudate activity by action contingency. Neuron, 41, 281-292.

Tversky, A., \& Kahneman, D. (1992). Advances in prospect theory: Cumulative representation of uncertainty. Journal of Risk and Uncertainty, 5, 297-323. 
Tymula, A., Rosenberg Belmaker, L. A., Roy, A. K., Ruderman, L., Manson, K., Glimcher, P. W., et al. (2012). Adolescents' risk-taking behavior is driven by tolerance to ambiguity. Proceedings of the National Academy of Sciences, U.S.A., 109, 17135-17140.

Tymula, A., Rosenberg Belmaker, L. A., Ruderman, L., Glimcher, P. W., \& Levy, I. (2013). Like cognitive function, decision making across the life span shows profound age-related changes. Proceedings of the National Academy of Sciences, U.S.A., 110, 17143-17148.

van Duijvenvoorde, A. C. K., Figner, B., Weeda, W. D., Van der Molen, M. W., Jansen, B. R., \& Huizenga, H. M. (2016).

Neural mechanisms underlying compensatory and noncompensatory strategies in risky choice. Journal of Cognitive Neuroscience, 28, 1358-1373.

van Duijvenvoorde, A. C. K., Huizenga, H. M., Somerville, L. H., Delgado, M. R., Powers, A., Weeda, W. D., et al. (2015).

Neural correlates of expected risks and returns in risky choice across development. Journal of Neuroscience, 35, 1549-1560.

van Duijvenvoorde, A. C. K., Peters, S., Braams, B. R., \& Crone, E. A. (2016). What motivates adolescents? Neural responses to rewards and their influence on adolescents' risk taking, learning, and cognitive control. Neuroscience \& Biobehavioral Reviews, 70, 135-147.
Volz, K. G., Schubotz, R. I., \& von Cramon, D. Y. (2003). Predicting events of varying probability: Uncertainty investigated by fMRI. Neuroimage, 19, 271-280.

Volz, K. G., Schubotz, R. I., \& von Cramon, D. Y. (2004). Why am I unsure? Internal and external attributions of uncertainty dissociated by fMRI. Neuroimage, 21, 848-857.

Von Gaudecker, H.-M., Van Soest, A., \& Wengström, E. (2011). Heterogeneity in risky choice behavior in a broad population. American Economic Review, 101, 664-694.

Woo, C.-W., Krishnan, A., \& Wager, T. D. (2014). Cluster-extent based thresholding in fMRI analyses: Pitfalls and recommendations. Neuroimage, 91, 412-419.

Yarkoni, T. (2009). Big correlations in little studies: Inflated fMRI correlations reflect low statistical power-Commentary on Vul et al. (2009). Perspectives on Psychological Science, 4, 294-298.

Yarkoni, T., Poldrack, R. A., Nichols, T. E., Van Essen, D. C., \& Wager, T. D. (2012). Large-scale automated synthesis of human functional neuroimaging data. Nature Methods, 8 , 665-670.

Yarkoni, T., Poldrack, R. A., Van Essen, D. C., \& Wager, T. D. (2010). Cognitive neuroscience 2.0: Building a cumulative science of human brain function. Trends in Cognitive Sciences, 14, 489-496. 